\title{
PARTIAL ACTIONS AND AN EMBEDDING THEOREM FOR INVERSE SEMIGROUPS
}

\author{
MYKOLA KHRYPCHENKO
}

\begin{abstract}
We give a simple construction involving partial actions which permits us to obtain an easy proof of a weakened version of L. O'Carroll's theorem on idempotent pure extensions of inverse semigroups.
\end{abstract}

\section{INTRODUCTION}

It was proved by D. B. McAlister in [9, Theorem 2.6] that, up to an isomorphism, each $E$-unitary inverse semigroup is of the form $P(G, X, Y)$ for some McAlister triple $(G, X, Y)$. There are several alternative proofs of this fact (see, for example, the survey [7]), in particular, the one given in [4] uses partial actions of groups on semilattices.

L. O'Carroll generalized in [12, Theorem 4] (which is a reformulation of [10, Theorem 2.11]) McAlister's $P$-theorem by showing that for any inverse semigroup $S$ and an idempotent pure congruence $\rho$ on $S$ there is a fully strict $L$-triple $(T, X, Y)$ with $T \cong S / \rho$ and $Y \cong E(S)$, such that $S \cong L_{m}(T, X, Y)$. This gives rise to a categorical equivalence as shown in [5, Theorems 1.5, 4.1 and 4.4]. Moreover, when $S$ is $E$-unitary and $\rho$ is the minimum group congruence on $S$, this coincides with McAlister's description of $S$ as $P(G, X, Y)$.

In this short note we prove the following weakened version of [12, Theorem 4]: given an inverse semigroup $S$ and an idempotent pure congruence $\rho$ on $S$, there exists a fully strict partial action $\tau$ of $S / \rho$ on $E(S)$, such that $S$ embeds into $E(S) \rtimes_{\tau}$ $(S / \rho)$. Moreover, the image of $S$ in $E(S) \rtimes_{\tau}(S / \rho)$ coincides with a subsemigroup $E(S) \rtimes_{\tau}^{m}(S / \rho)$ of $E(S) \rtimes_{\tau}(S / \rho)$ which can be explicitly described. Our approach permits one to avoid the necessity of the poset $X$ in O'Carroll's $L$-triple. On the other hand, this weakens the result, as the partial action $\tau$ that we construct may in fact be non-globalizable.

\section{Preliminaries}

Given an inverse semigroup $S$, we use the standard notation $\leq$ for the natural partial order on $S$, and $\wedge, \vee$ for the corresponding meet and join. Notice that $s \wedge t$ or $s \vee t$ may not exist in $S$ in general. However, if $e$ and $f$ are idempotents of $S$, then $e \wedge f$ exists and coincides with $e f$, which is again an idempotent. Thus, the subset of idempotents of $S$, denoted by $E(S)$, forms a (meet) semilattice with respect to $\wedge$.

2010 Mathematics Subject Classification. Primary 20M18, 20M30; Secondary 20M15.

Key words and phrases. Inverse semigroup, premorphism, idempotent pure congruence.

Supported by FAPESP of Brazil (process number: 2012/01554-7). 
A congruence $\rho$ on $S$ is said to be idempotent pure, if

$$
(e, s) \in \rho \& e \in E(S) \Rightarrow s \in E(S) .
$$

The semigroup $S$ is called E-unitary, if its minimum group congruence $\sigma$, defined by

$$
(s, t) \in \sigma \Leftrightarrow \exists u \leq s, t,
$$

is idempotent pure. An $F$-inverse monoid is an inverse semigroup, in which every $\sigma$-class has a maximum element under $\leq$. Each $F$-inverse monoid is $E$-unitary (see [6, Proposition 7.1.3]).

Given a set $X$, by $\mathcal{I}(X)$ we denote the symmetric inverse monoid on $X$. The elements of $\mathcal{I}(X)$ are the partial bijections of $X$, and the product $f g$ of $f, g \in \mathcal{I}(X)$ is the composition, i.e.

$$
f g: g^{-1}(\operatorname{dom} f \cap \operatorname{ran} g) \rightarrow f(\operatorname{dom} f \cap \operatorname{ran} g),(f g)(x)=f(g(x)) .
$$

Observe using [6. Proposition 1.1.4 (3)] that $f \leq g$ in $\mathcal{I}(X)$ if and only if $f \subseteq g$ regarded as subsets of $X \times X$. It follows that

$$
\text { there exists } \bigvee_{i \in I} f_{i} \text { in } \mathcal{I}(X) \Leftrightarrow \bigcup_{i \in I} f_{i} \in \mathcal{I}(X) \text {, in which case } \bigvee_{i \in I} f_{i}=\bigcup_{i \in I} f_{i} \text {. }
$$

Here $\cup$ is the union of functions as subsets of $X \times X$.

Let $S$ and $T$ be inverse semigroups. Recall from [4] that a map $\tau: S \rightarrow T$ is called a premorphism 1 , if

(i) $\tau\left(s^{-1}\right)=\tau(s)^{-1}$;

(ii) $\tau(s) \tau(t) \leq \tau(s t)$.

One can easily show that

$$
\tau(E(S)) \subseteq E(T)
$$

(see, for example, 14]). By a partial action of an inverse semigroup $S$ on a set $X$ we mean a premorphism $\tau: S \rightarrow \mathcal{I}(X)$. If $\tau$ is a homomorphism, then we say that the partial action $\tau$ is global. We use the notation $\tau_{s}$ for the partial bijection $\tau(s)$.

\section{Construction}

We fist make several observations. By [6, Proposition 2.4.5] a congruence $\rho$ on $S$ is idempotent pure if and only if

$$
\rho \subseteq \sim,
$$

where $\sim$ is the compatibility relation on $S$ defined by

$$
s \sim t \Leftrightarrow s^{-1} t, s t^{-1} \in E(S) .
$$

Moreover, $\sim \subseteq \sigma$ by [6, Theorem 2.4.1 (1)], whence

$$
\rho \subseteq \sigma
$$

for each idempotent pure congruence $\rho$ on $S$.

We would also like to note that, given a partial action $\tau$ of $S$ on $X$ and $s \in S$, then by (i) and (ii)

$$
\operatorname{id}_{\mathrm{ran} \tau_{s}}=\tau_{s} \tau_{s}^{-1}=\tau_{s} \tau_{s^{-1}} \leq \tau_{s s^{-1}} .
$$

\footnotetext{
${ }^{1}$ In 13 such a map is called a prehomomorphism, and in 86 14] it is called a dual prehomomorphism.
} 
Lemma 2.1. Let $S$ be an inverse semigroup, $X$ a set, $\rho$ an idempotent pure congruence on $S$ and $\tau$ a partial action of $S$ on $X$. Then for any $\rho$-class $[s] \in S / \rho$ the join $\bigvee_{t \in[s]} \tau_{t}$ exists in $\mathcal{I}(X)$.

Proof. Let $(s, t) \in \rho$. Then by (i) and (ii) one has that $\tau_{s} \tau_{t}^{-1}=\tau_{s} \tau_{t^{-1}} \leq \tau_{s t^{-1}}$ and similarly $\tau_{s}^{-1} \tau_{t} \leq \tau_{s^{-1} t}$. Since $\tau_{s t^{-1}}, \tau_{s^{-1} t} \in E(\mathcal{I}(X))$ thanks to (2), (3) and (4), it follows from [6. Proposition 1.2.1 (2)] that $\tau_{s} \cup \tau_{t} \in \mathcal{I}(X)$. It remains to apply [6, Proposition 1.2.1 (3)] and (11).

Lemma 2.2. Under the conditions of Lemma[2.1] define $\tilde{\tau}: S / \rho \rightarrow \mathcal{I}(X),[s] \mapsto \tilde{\tau}_{[s]}$, by

$$
\tilde{\tau}_{[s]}=\bigvee_{t \in[s]} \tau_{t} .
$$

Then $\tilde{\tau}$ is a partial action of $S / \rho$ on $X$.

Proof. By Lemma 2.1 the map $\tilde{\tau}$ is well defined. Using [6, Proposition 1.2.1 (4)] and (i) we obtain that

$$
\tilde{\tau}_{[s]}^{-1}=\bigvee_{t \in[s]} \tau_{t}^{-1}=\bigvee_{t \in[s]} \tau_{t^{-1}}=\bigvee_{t \in\left[s^{-1}\right]} \tau_{t}=\tilde{\tau}_{\left[s^{-1}\right]}=\tilde{\tau}_{[s]^{-1}}
$$

Moreover, applying [6. Proposition 1.2.1 (5)] and (ii)] we conclude that

$$
\begin{aligned}
\tilde{\tau}_{[s]} \tilde{\tau}_{[t]} & =\left(\bigvee_{s^{\prime} \in[s]} \tau_{s^{\prime}}\right)\left(\underset{t^{\prime} \in[t]}{\bigvee} \tau_{t^{\prime}}\right)=\bigvee_{s^{\prime} \in[s]} \tau_{s^{\prime}}\left(\bigvee_{t^{\prime} \in[t]} \tau_{t^{\prime}}\right)=\bigvee_{s^{\prime} \in[s], t^{\prime} \in[t]} \tau_{s^{\prime}} \tau_{t^{\prime}} \\
& \leq \bigvee_{s^{\prime} \in[s], t^{\prime} \in[t]} \tau_{s^{\prime} t^{\prime}} \leq \bigvee_{u \in[s t]} \tau_{u}=\tilde{\tau}_{[s t]}=\tilde{\tau}_{[s][t]} .
\end{aligned}
$$

Remark 2.3. If, under the conditions of Lemma 2.2. $S$ is E-unitary, $\rho=\sigma$ and $\bigcup_{e \in E(S)} \operatorname{dom} \tau_{e}=X$, then $\tilde{\tau}$ is a partial action [2, 4] of the maximum group image $\mathcal{G}(S)=S / \sigma$ of $S$ on $X$ as in [14, Theorem 1.2].

Remark 2.4. If, under the conditions of Lemma $2.2, S$ is an $F$-inverse monoid, $\tau$ is global and $\rho=\sigma$, then $\tilde{\tau}_{[s]}=\tau_{\max [s]}$. In particular, if $G$ is a group, $S$ is the monoid $\mathcal{S}(G)$ from $[2]^{2}$ and $\tau_{1_{S}}=\operatorname{id}_{X}$, then identifying $\mathcal{G}(S)$ with $G$, we see that $\tilde{\tau}$ is the partial action of $G$ on $X$ induced by $\tau$ as in [2, Theorem 4.2].

Let $(E, \leq)$ be a meet semilattice, i.e. a partially ordered set, in which any pair of elements $e, f \in E$ has a meet $e \wedge f$. Then $(E, \wedge)$ is a commutative semigroup, and all its elements are idempotents. It follows that $E$ is inverse, and the natural partial order on $E$ coincides with $\leq$. The converse is also true: each commutative inverse semigroup $S$, such that $E(S)=S$, is a meet semilattice, where $e \wedge f=e f$ for all $e, f \in S$ (see [6. Proposition 1.4.9]). Notice that an order ideal of $(E, \leq)$ is a semigroup ideal of $(E, \wedge)$, an order isomorphism $\left(E_{1}, \leq_{1}\right) \rightarrow\left(E_{2}, \leq_{2}\right)$ is a semigroup isomorphism $\left(E_{1}, \wedge_{1}\right) \rightarrow\left(E_{2}, \wedge_{2}\right)$, and vice versa, so we may use the terms 'ideal' and 'isomorphism' in both senses.

Following [13] for any meet semilattice $E$ we denote by $\Sigma(E)$ the inverse subsemigroup of $\mathcal{I}(E)$ consisting of the isomorphisms between ideals of $E$. Given an

\footnotetext{
${ }^{2}$ Notice that $\mathcal{S}(G)$ coincides with Birget-Rhodes prefix expansion of $G$ (see 1,15$]$ )
} 
inverse semigroup $S$, by a partial action $\tau$ of $S$ on $E$ we mean a premorphism $\tau: S \rightarrow \Sigma(E)$. For any such $\tau$ set

$$
E \rtimes_{\tau} S=\left\{(e, s) \in E \times S \mid e \in \operatorname{ran} \tau_{s}\right\}
$$

with the multiplication

$$
(e, s)(f, t)=\left(\tau_{s}\left(\tau_{s}^{-1}(e) \wedge f\right), s t\right) .
$$

Since $\tau_{s}\left(\tau_{s}^{-1}(e) \wedge f\right) \in \tau_{s}\left(\operatorname{dom} \tau_{s} \cap \operatorname{ran} \tau_{t}\right)=\operatorname{ran}\left(\tau_{s} \tau_{t}\right) \subseteq \operatorname{ran} \tau_{s t}$, the product (9) is well defined.

Lemma 2.5. The set $E \rtimes_{\tau} S$ is an inverse semigroup with respect to the operation (91). Its semilattice of idempotents is $\left\{(e, f) \in E \rtimes_{\tau} S \mid f \in E(S)\right\}$ and $(e, s)^{-1}=\left(\tau_{s}^{-1}(e), s^{-1}\right)$ for every $(e, s) \in E \rtimes_{\tau} S$.

Proof. See [13, Lemmas VI.7.6-7].

Lemma 2.6. Let $\tau$ be a partial action of an inverse semigroup $S$ on a meet semilattice $E$ and $\rho$ an idempotent pure congruence on $S$. Then $\tilde{\tau}$ is a partial action of $S / \rho$ on $E$.

Proof. We know from Lemma 2.2 that $\tilde{\tau}$ is a premorphism from $S / \rho$ to $\mathcal{I}(E)$. So, we only need to prove that $\tilde{\tau}(S / \rho) \subseteq \Sigma(E)$. Clearly, dom $\tilde{\tau}_{[s]}$ and $\operatorname{ran} \tilde{\tau}_{[s]}$ are ideals of $E$ as unions of ideals dom $\tau_{t}$ and $\operatorname{ran} \tau_{t}$, respectively, where $t \in[s]$. Furthermore, let $e, f \in \operatorname{dom} \tilde{\tau}_{[s]}$ and $e \leq f$. There exists $t \in[s]$, such that $f \in \operatorname{dom} \tau_{t}$. Since $\operatorname{dom} \tau_{t}$ is an ideal, it follows that $e \in \operatorname{dom} \tau_{t}$. Then, taking into account the fact that $\tau_{t}$ preserves $\leq$, we have

$$
\tilde{\tau}_{[s]}(e)=\tau_{t}(e) \leq \tau_{t}(f)=\tilde{\tau}_{[s]}(f) .
$$

Since $\tilde{\tau}_{[s]}^{-1}=\tilde{\tau}_{[s]^{-1}}$, then $\tilde{\tau}_{[s]}^{-1}$ is also order-preserving. Thus, $\tilde{\tau}_{[s]} \in \Sigma(E)$.

\section{The EMBEDding THEOREM}

Let $S$ be an inverse semigroup. It is well known (see, for example, 6, Theorem 5.2.8]) that the map $\delta: S \rightarrow \Sigma(E(S)), s \mapsto \delta_{s}$, where

$$
\delta_{s}: s^{-1} s E(S) \rightarrow s s^{-1} E(S) \text { and } \delta_{s}(e)=s e s^{-1} \text { for any } e \in s^{-1} s E(S),
$$

is a global action of $S$ on $E(S)$, called the Munn representation of $S$.

Proposition 3.1. Let $S$ be an inverse semigroup and $\rho$ an idempotent pure congruence on $S$. Then $S$ embeds into $E(S) \rtimes_{\tilde{\delta}}(S / \rho)$.

Proof. Define $\varphi: S \rightarrow E(S) \rtimes_{\tilde{\delta}}(S / \rho)$ by

$$
\varphi(s)=\left(s s^{-1},[s]\right) .
$$

As $s s^{-1} \in s s^{-1} E(S) \subseteq \operatorname{ran} \tilde{\delta}_{[s]}$, the map $\varphi$ is well defined. Since by (7) and (10)

$$
\begin{aligned}
\left(s s^{-1},[s]\right)\left(t t^{-1},[t]\right) & =\left(\tilde{\delta}_{[s]}\left(\tilde{\delta}_{[s]^{-1}}\left(s s^{-1}\right) t t^{-1}\right),[s t]\right) \\
& =\left(\tilde{\delta}_{[s]}\left(\delta_{s^{-1}}\left(s s^{-1}\right) t t^{-1}\right),[s t]\right) \\
& =\left(\tilde{\delta}_{[s]}\left(s^{-1} s \cdot t t^{-1}\right),[s t]\right) \\
& =\left(\delta_{s}\left(s^{-1} s \cdot t t^{-1}\right),[s t]\right) \\
& =\left(s t t^{-1} s^{-1},[s t]\right) \\
& =\left(s t(s t)^{-1},[s][t]\right),
\end{aligned}
$$


$\varphi$ is a homomorphism. Suppose that $\varphi(s)=\varphi(t)$. By (11) this means that $(s, t) \in$ $\mathcal{R} \cap \rho$, where $\mathcal{R}$ is the Green's relation on $S$, defined by

$$
(s, t) \in \mathcal{R} \Leftrightarrow s s^{-1}=t t^{-1} .
$$

As $\mathcal{R} \cap \rho \subseteq \mathcal{R} \cap \sim$, it follows by [13, Lemma III.2.13] that $s=t$, and hence $\varphi$ is one-to-one.

To make the result of Proposition 3.1 more precise, we follow [12. We say that a partial action $\tau$ of $S$ on $E$ is strict, if for each $e \in E$ the set $\{f \in E(S) \mid e \in$ $\left.\operatorname{dom} \tau_{f}\right\}$ has a minimum element, denoted by $\alpha(e)$, and the map $\alpha: E \rightarrow E(S)$ is a homomorphism of meet semilattices. Observe using (6) that if $(e, s) \in E \rtimes_{\tau} S$, then $\alpha(e) \leq s s^{-1}$. The set of $(e, s) \in E \rtimes_{\tau} S$ for which

$$
\alpha(e)=s s^{-1}
$$

will be denoted by $E \rtimes_{\tau}^{m} S$.

Lemma 3.2. The subset $E \rtimes_{\tau}^{m} S$ is an inverse subsemigroup of $E \rtimes_{\tau} S$.

Proof. We first observe, as in [12, Lemma 2], that

$$
\alpha\left(\tau_{s}(e)\right)=s \alpha(e) s^{-1}
$$

for any $e \in \operatorname{dom} \tau_{s}$. Indeed, $e \in \operatorname{dom} \tau_{\alpha(e)}$, therefore using (ii)] and (6) we have

$$
\tau_{s}(e) \in \operatorname{ran}\left(\tau_{s} \tau_{\alpha(e)}\right) \subseteq \operatorname{ran} \tau_{s \alpha(e)} \subseteq \operatorname{dom} \tau_{s \alpha(e)(s \alpha(e))^{-1}}=\operatorname{dom} \tau_{s \alpha(e) s^{-1}},
$$

whence

$$
\alpha\left(\tau_{s}(e)\right) \leq s \alpha(e) s^{-1} .
$$

For the converse inequality replace $e$ by $\tau_{s}(e)$ and $s$ by $s^{-1}$ in (14) and then use (i)

Let $(e, s),(f, t) \in E \rtimes_{\tau}^{m} S$. Then for their product $\left(\tau_{s}\left(\tau_{s}^{-1}(e) \wedge f\right), s t\right)$ we have by (i), (12) and (13) that

$$
\alpha\left(\tau_{s}\left(\tau_{s}^{-1}(e) \wedge f\right)\right)=s \alpha\left(\tau_{s}^{-1}(e) \wedge f\right) s^{-1}=s \cdot s^{-1} \alpha(e) s \cdot \alpha(f) s^{-1}=s t t^{-1} s^{-1} .
$$

Hence, $(e, s)(f, t) \in E \rtimes_{\tau}^{m} S$. Moreover, for each $(e, s) \in E \rtimes_{\tau}^{m} S$ the inverse $(e, s)^{-1}=\left(\tau_{s}^{-1}(e), s^{-1}\right)$ belongs to $E \rtimes_{\tau}^{m} S$, as $\alpha\left(\tau_{s}^{-1}(e)\right)=s^{-1} \alpha(e) s=s^{-1} s$.

A strict partial action $\tau$ of $S$ on $E$ is called fully strict, if the homomorphism

$$
\pi: E \rtimes_{\tau}^{m} S \rightarrow S,(e, s) \mapsto s,
$$

is surjective.

Remark 3.3. Let $\tau$ be a fully strict partial action of $S$ on $E$. Then $E \rtimes_{\tau}^{m} S=E \rtimes_{\tau} S$ if and only if $S$ is a group.

Indeed, the "if" part trivially holds for any strict $\tau$. For the "only if" part take $s \in S$ and suppose that $s \leq t$ for some $t \in S$. Since $\tau$ is fully strict, there are $e, f \in E$, such that $(e, s),(f, t) \in E \rtimes_{\tau}^{m} S$. In particular, $e \in \operatorname{ran} \tau_{s}$ and $f \in \operatorname{ran} \tau_{t}$. Since $\operatorname{ran} \tau_{s}$ and $\operatorname{ran} \tau_{t}$ are ideals of $E$, we have $e f \in \operatorname{ran} \tau_{s} \cap \operatorname{ran} \tau_{t}$, whence $(e f, s),(e f, t) \in E \rtimes_{\tau} S$. But $E \rtimes_{\tau} S=E \rtimes_{\tau}^{m} S$, so $s s^{-1}=\alpha(e f)=t t^{-1}$ by (12). Consequently, $(s, t) \in \mathcal{R}$. Then $s=t$ thanks to [6. Proposition 3.2 .3 (2)], and thus the natural partial order on $S$ is trivial. It remains to apply [6, Proposition 1.4.10]. 
Theorem 3.4. Given an inverse semigroup $S$ and an idempotent pure congruence $\rho$ on $S$, there exists a fully strict partial action $\tau$ of $S / \rho$ on $E(S)$, such that $S$ embeds into $E(S) \rtimes_{\tau}(S / \rho)$. Moreover, the image of $S$ in $E(S) \rtimes_{\tau}(S / \rho)$ coincides with $E(S) \rtimes_{\tau}^{m}(S / \rho)$, and $\rho$ is induced by the epimorphism $\pi: E(S) \rtimes_{\tau}^{m}(S / \rho) \rightarrow S / \rho$. In particular, the embedding is surjective if and only if $\rho$ is a group congruence, i.e. $S$ is E-unitary and $\rho=\sigma$.

Proof. As we know from Proposition 3.1 there is an embedding $\varphi: S \rightarrow E(S) \rtimes_{\tilde{\delta}}$ $(S / \rho)$ given by (11). For each $e \in E(S)$ consider the set

$$
A_{e}=\left\{[f] \in E(S / \rho) \mid e \in \operatorname{dom} \tau_{[f]}\right\} .
$$

Clearly, $[e] \in A_{e}$, as $e \in e E(S)=\operatorname{dom} \delta_{e} \subseteq \operatorname{dom} \tilde{\delta}_{[e]}$. Moreover, if $[f] \in A_{e}$, then $e \in$ $g E(S)$ for some idempotent $g \in[f]$. So, $e \leq g$ and hence $[e] \leq[g]=[f]$. This shows that $[e]$ is the minimum element of $A_{e}$, and since $\alpha(e)=[e]$ is a homomorphism $E(S) \rightarrow E(S / \rho)$, the partial action $\tilde{\delta}$ is strict. Now, for each $s \in S$ consider the pair $\varphi(s)=\left(s s^{-1},[s]\right) \in E(S) \rtimes_{\tilde{\delta}}(S / \rho)$. We have $\alpha\left(s s^{-1}\right)=\left[s s^{-1}\right]=[s][s]^{-1}$, so in fact $\left(s s^{-1},[s]\right) \in E(S) \rtimes_{\tilde{\delta}}^{m}(S / \rho)$, proving that $\tilde{\delta}$ is fully strict.

The range of the embedding $\varphi$ is contained in $E(S) \rtimes_{\tilde{\delta}}^{m}(S / \rho)$ as shown above. Now if $(e,[s]) \in E(S) \rtimes_{\tilde{\delta}}^{m}(S / \rho)$, then $e \in t t^{-1} E(S)$ for some $t \in[s]$ and $[e]=\alpha(e)=$ $[s][s]^{-1}$. It follows that $e=e t t^{-1}=e t(e t)^{-1}$ and $[e t]=[s][s]^{-1}[t]=[s][s]^{-1}[s]=[s]$. Therefore, $(e,[s])=\varphi(e t)$, whence $\varphi(S)=E(S) \rtimes_{\tilde{\delta}}^{m}(S / \rho)$. Moreover, $\pi(\varphi(s))=$ $\pi(\varphi(t)) \Leftrightarrow[s]=[t] \Leftrightarrow(s, t) \in \rho$ in view of (11) and (15).

Finally, if $\varphi$ is surjective, then $E(S) \rtimes_{\tilde{\delta}}^{m}(S / \rho)=E(S) \rtimes_{\tilde{\delta}}(S / \rho)$, so $S / \rho$ is a group by Remark 3.3. Therefore, $\rho \supseteq \sigma$ by [6, Theorem 2.4.1 (3)], which yields $\rho=\sigma$ in view of 5 , and thus $S$ is $E$-unitary.

\section{Globalizable partial actions and O'Carroll $L$-triples}

Let $S$ be an inverse semigroup, $\phi: S \rightarrow \mathcal{I}(X), s \mapsto \phi_{s}$, a partial action of $S$ on a set $X$ and $Y \subseteq X$. Then for any $s \in S$ the partial bijection

$$
\tau_{s}=\operatorname{id}_{Y} \phi_{s} \operatorname{id}_{Y}
$$

is the restriction of $\phi_{s}$ to the subset

$$
\operatorname{dom} \tau_{s}=\left\{y \in \operatorname{dom} \phi_{s} \cap Y \mid \phi_{s}(y) \in Y\right\}=\phi_{s}^{-1}\left(\operatorname{ran} \phi_{s} \cap Y\right) \cap Y \subseteq \operatorname{dom} \phi_{s} .
$$

Thus, $\tau_{s}$ is a bijection

$$
\phi_{s}^{-1}\left(\operatorname{ran} \phi_{s} \cap Y\right) \cap Y \rightarrow \phi_{s}\left(\operatorname{dom} \phi_{s} \cap Y\right) \cap Y .
$$

Moreover,

$$
\tau_{s} \tau_{t}=\operatorname{id}_{Y} \phi_{s} \operatorname{id}_{Y} \phi_{t} \operatorname{id}_{Y} \leq \operatorname{id}_{Y} \phi_{s} \phi_{t} \operatorname{id}_{Y} \leq \operatorname{id}_{Y} \phi_{s t} \operatorname{id}_{Y}=\tau_{s t},
$$

so that $\tau: S \rightarrow \mathcal{I}(Y), s \mapsto \tau_{s}$, is a partial action of $S$ on $Y$ called the restriction of $\phi$ to $Y$.

A partial action $\tau$ of an inverse semigroup $S$ on a set $Y$ is said to be globalizable if it admits a globalization 3 , i.e. a global action $\phi$ of $S$ on a set $X$ together with an injective map $\iota: Y \rightarrow X$, such that

$$
\tau_{s}=\bar{\iota} \phi_{s} \bar{\iota}^{-1}
$$

\footnotetext{
${ }^{3}$ In a more general context this was called an augmented action in 3
} 
holds for all $s \in S$. Here $\bar{\iota}$ is the bijection $Y \rightarrow \iota(Y), y \mapsto \iota(y)$. It was proved in [3, Theorem 6.10] that $\tau$ is globalizable provided that it is order-preserving, i.e. $s \leq t \Rightarrow \tau_{s} \leq \tau_{t}$ for all $s, t \in S$. Observe that the converse of this fact is also true. For, a global action $\phi$ of $S$ on $X$, being a homomorphism $S \rightarrow \mathcal{I}(X)$, is order-preserving, so that its restriction $\tau$ defined by (16) is also order-preserving.

Globalizable partial actions of inverse semigroups on semilattices turn out to be closely related to O'Carroll $L$-triples. Recall from [12] that an $L$-triple is $(T, X, Y)$, where

(i) $T$ is an inverse semigroup, $X$ is a down-directed poset, $Y$ is a meet subsemilattice and order ideal of $X$;

(ii) $T$ acts (globally) on $X$ via a homomorphism $\phi: T \rightarrow \mathcal{I}(X), t \mapsto \phi_{t}$, such that $\phi_{t}$ is an order isomorphism between non-empty order ideals of $X$;

(iii) $X=T Y$, where $T Y=\bigcup_{t \in T} \phi_{t}\left(\operatorname{dom} \phi_{t} \cap Y\right)$.

With any $L$-triple $(T, X, Y) \mathrm{O}^{\prime}$ Carroll associates in 12 the inverse semigroup

$$
L(T, X, Y)=\left\{(a, t) \in X \times T \mid a \in Y \cap \operatorname{ran} \phi_{t}, \phi_{t}^{-1}(a) \in Y\right\},
$$

where the product of any two pairs from $L(T, X, Y)$ is given by the same formula (9) as we used in the case of a partial action. An $L$-triple $(T, X, Y)$ was called strict in [12] if for every $y \in Y$ the set $\left\{f \in E(T) \mid y \in \operatorname{dom} \phi_{f}\right\}$ has a minimum element $e(y)$, and the map $e: Y \rightarrow E(T), y \mapsto e(y)$, is a homomorphism of meet semilattices. In this case

$$
L_{m}(T, X, Y)=\left\{(a, t) \in L(T, X, Y) \mid e(a)=t t^{-1}\right\}
$$

is an inverse subsemigroup of $L(T, X, Y)$ by [12, Theorem 2]. Moreover, if the homomorphism $L_{m}(T, X, Y) \rightarrow T,(a, t) \mapsto t$, is surjective, then the strict $L$-triple $(T, X, Y)$ was said to be fully strict [12].

Proposition 4.1. Let $(T, X, Y)$ be an L-triple and $\phi: T \rightarrow \mathcal{I}(X)$ the corresponding action of $T$ on $X$. Denote by $\tau$ the restriction of $\phi$ to $Y$. Then $\tau$ is a partial action of $T$ on the meet semilattice $Y$ such that $\operatorname{dom} \tau_{t} \neq \emptyset$ for all $t \in T$, and $L(T, X, Y)=Y \rtimes_{\tau} T$. Moreover, $\tau$ is strict (resp. fully strict) if and only if $(T, X, Y)$ is strict (resp. fully strict), in which case $L_{m}(T, X, Y)=Y \rtimes_{\tau}^{m} T$.

Proof. It was shown in [11, Lemma 3] that, given an action $\phi$ of $T$ on $(X, \leq)$, such that $\phi_{t}$ is an order isomorphism between order ideals of $X$ for all $t \in T$, and an order ideal $Y$ of $X$ satisfying $T Y=X$, one has that

$X$ is down-directed \& $\forall t \in T: \operatorname{dom} \phi_{t} \neq \emptyset \Leftrightarrow \forall t \in T: \phi_{t}\left(\operatorname{dom} \phi_{t} \cap Y\right) \cap Y \neq \emptyset$.

By (17) the latter is the same as $\forall t \in T: \operatorname{ran} \tau_{t} \neq \emptyset$ or, equivalently, $\forall t \in T$ : $\operatorname{dom} \tau_{t} \neq \emptyset$. Let $y \in \operatorname{dom} \tau_{t}$, i.e. $y \in \operatorname{dom} \phi_{t} \cap Y$ and $\phi_{t}(y) \in Y$. For any $z \leq y$ one has that $z \in \operatorname{dom} \phi_{t} \cap Y$, as $Y$ and $\operatorname{dom} \phi_{t}$ are ideals of $X$. Moreover, $\phi_{t}(z) \leq \phi_{t}(y) \in Y$ since $\phi_{t}$ is order-preserving, so $\phi_{t}(z) \in Y$. Thus, $z \in \operatorname{dom} \tau_{t}$, and consequently $\tau_{t} \in \Sigma(Y)$ for all $t \in T$.

Notice using (17) and (19) that $(a, t) \in L(T, X, Y) \Leftrightarrow a \in \operatorname{ran} \tau_{t}$. Thus, $L(T, X, Y)$ is exactly $Y \rtimes_{\tau} T$ defined by (8). Now, for any $y \in Y$ and $f \in E(T)$

$$
y \in \operatorname{dom} \phi_{f} \Leftrightarrow y \in \operatorname{dom} \phi_{f} \cap Y=\phi_{f}\left(\operatorname{dom} \phi_{f} \cap Y\right) \cap Y=\operatorname{dom} \tau_{f} .
$$

Hence, $(T, X, Y)$ is strict if and only if $\tau$ is strict. The remaining assertions of the proposition are immediate. 
Lemma 4.2. Let $\tau$ be a strict partial action of an inverse semigroup $T$ on a semilattice $(Y, \leq)$. If $\tau$ is globalizable, then $\tau$ admits a globalization $\phi: T \rightarrow \mathcal{I}(X)$ such that $T \iota(Y)=X$, where $\iota: Y \rightarrow X$ is the corresponding injective map.

Proof. Let $\phi^{\prime}: T \rightarrow \mathcal{I}\left(X^{\prime}\right), \iota: Y \rightarrow X^{\prime}$ be a globalization of $\tau$. Without loss of generality we may identify $Y$ with $\iota(Y)$, so that $Y \subseteq X^{\prime}, \iota: Y \rightarrow X^{\prime}$ is the inclusion map and $\tau$ is the restriction of $\phi^{\prime}$ to $Y$. Consider

$$
X=T Y \subseteq X^{\prime} \text { and } \phi \text { being the restriction of } \phi^{\prime} \text { to } X \text {. }
$$

Since $\tau$ is strict, each $y \in Y$ belongs to

$$
\operatorname{dom} \tau_{\alpha(y)}=\operatorname{ran} \tau_{\alpha(y)}=\phi_{\alpha(y)}^{\prime}\left(\operatorname{dom} \phi_{\alpha(y)}^{\prime} \cap Y\right) \cap Y \subseteq X,
$$

so $Y \subseteq X$. Moreover, notice by (21) that

$$
x \in \operatorname{dom} \phi_{t}^{\prime} \cap X \Rightarrow \phi_{t}^{\prime}(x) \in X .
$$

Indeed, if $x \in \operatorname{dom} \phi_{t}^{\prime}$ and $x=\phi_{u}^{\prime}(y)$ for some $u \in T$ and $y \in \operatorname{dom} \phi_{u}^{\prime} \cap Y$, then $\phi_{t}^{\prime}(x)=\phi_{t u}^{\prime}(y)$. This implies that

$$
\operatorname{dom} \phi_{t}=\operatorname{dom} \phi_{t}^{\prime} \cap X \text {. }
$$

It follows that (18) holds, and we only need to show that the partial action $\phi$ is global, i.e. $\phi_{t u}=\phi_{t} \phi_{u}$ for all $t, u \in T$. Using the fact that $\phi^{\prime}$ is global, (22) and (23), we have

$$
\begin{aligned}
y & \in \operatorname{dom} \phi_{t u}=\operatorname{dom} \phi_{t u}^{\prime} \cap X=\operatorname{dom} \phi_{t}^{\prime} \phi_{u}^{\prime} \cap X \\
& \Leftrightarrow y \in \operatorname{dom} \phi_{u}^{\prime} \cap X \& \phi_{u}^{\prime}(y) \in \operatorname{dom} \phi_{t}^{\prime} \\
& \Leftrightarrow y \in \operatorname{dom} \phi_{u}^{\prime} \cap X \& \phi_{u}^{\prime}(y) \in \operatorname{dom} \phi_{t}^{\prime} \cap X \\
& \Leftrightarrow y \in \operatorname{dom} \phi_{u} \& \phi_{u}(y) \in \operatorname{dom} \phi_{t} \\
& \Leftrightarrow y \in \operatorname{dom} \phi_{t} \phi_{u},
\end{aligned}
$$

in which case $\phi_{t u}(y)=\phi_{t u}^{\prime}(y)=\phi_{t}^{\prime}\left(\phi_{u}^{\prime}(y)\right)=\phi_{t}\left(\phi_{u}(y)\right)$.

Lemma 4.3. Let $\tau$ be a strict partial action of an inverse semigroup $T$ on a semilattice $(Y, \leq)$ and $\phi: T \rightarrow \mathcal{I}(X)$ a globalization of $\tau$ such that $T \iota(Y)=X$, where $\iota: Y \rightarrow X$ is the corresponding injective map. Then there exists a partial order $\leq^{\prime}$ on $X$, such that $\phi_{t}: \operatorname{dom} \phi_{t} \rightarrow \operatorname{ran} \phi_{t}$ is an order isomorphism between order ideals of $X$. Moreover, $\left(\iota(Y), \leq^{\prime}\right)$ is an order ideal and meet subsemilattice of $\left(X, \leq^{\prime}\right)$ isomorphic to $(Y, \leq)$.

Proof. As in the proof of Lemma 4.2 we assume for simplicity that $Y \subseteq X$ and $\iota: Y \rightarrow X$ is the inclusion map. Define the relation $\leq^{\prime}$ on $X$ as follows

$$
\begin{gathered}
x_{1} \leq^{\prime} x_{2} \Leftrightarrow \exists t \in T \text { and } \exists y_{1}, y_{2} \in \operatorname{dom} \phi_{t} \cap Y \text { such that } \\
x_{1}=\phi_{t}\left(y_{1}\right), x_{2}=\phi_{t}\left(y_{2}\right) \text { and } y_{1} \leq y_{2} .
\end{gathered}
$$

Reflexivity of $\leq^{\prime}$ is explained by the fact that $T Y=X$. For anti-symmetry suppose that $x_{1} \leq^{\prime} x_{2}$ and $x_{2} \leq^{\prime} x_{1}$, i.e. $x_{1}=\phi_{t}\left(y_{1}\right)=\phi_{u}\left(z_{1}\right)$ and $x_{2}=\phi_{t}\left(y_{2}\right)=\phi_{u}\left(z_{2}\right)$ for some $t, u \in T$ and $y_{1}, y_{2} \in \operatorname{dom} \phi_{t} \cap Y, z_{1}, z_{2} \in \operatorname{dom} \phi_{u} \cap Y$, such that $y_{1} \leq y_{2}$ and $z_{2} \leq z_{1}$. Then $z_{1}=\phi_{u}^{-1}\left(\phi_{t}\left(y_{1}\right)\right)=\phi_{u^{-1} t}\left(y_{1}\right)=\tau_{u^{-1}}\left(y_{1}\right)$ and similarly $z_{2}=$ $\tau_{u^{-1} t}\left(y_{2}\right)$. Since $y_{1} \leq y_{2}$ and $\tau_{u^{-1} t}$ is order-preserving, we conclude that $z_{1} \leq z_{2}$, whence $z_{1}=z_{2}$ by anti-symmetry of $\leq$, and thus $x_{1}=x_{2}$. For transitivity of $\leq^{\prime}$ 
take $x_{1} \leq^{\prime} x_{2}$ and $x_{2} \leq^{\prime} x_{3}$. By (24) there are $t, u \in T$ and $y_{1}, y_{2} \in \operatorname{dom} \phi_{t} \cap Y$, $z_{1}, z_{2} \in \operatorname{dom} \phi_{u} \cap Y$, such that

$$
\begin{aligned}
& x_{1}=\phi_{t}\left(y_{1}\right), \\
& x_{2}=\phi_{t}\left(y_{2}\right)=\phi_{u}\left(z_{1}\right), \\
& x_{3}=\phi_{u}\left(z_{2}\right)
\end{aligned}
$$

and $y_{1} \leq y_{2}, z_{1} \leq z_{2}$. It follows from (26) that $y_{2}=\phi_{t^{-1} u}\left(z_{1}\right)=\tau_{t^{-1} u}\left(z_{1}\right)$. Since $\operatorname{ran} \tau_{t^{-1} u}$ is an ideal of $Y$ and $y_{1} \leq y_{2}$, one has that $y_{1}=\tau_{t^{-1} u}\left(z_{0}\right)$ for some $z_{0} \in \operatorname{dom} \tau_{t^{-1} u}$. Applying $\tau_{t^{-1} u}^{-1}$ to the inequality $y_{1} \leq y_{2}$, we obtain $z_{0} \leq z_{1}$, whence $z_{0} \leq z_{2}$ by transitivity of $\leq$. Using (25), we have $\phi_{u}\left(z_{0}\right)=\phi_{u}\left(\tau_{t^{-1} u}^{-1}\left(y_{1}\right)\right)=$ $\phi_{u}\left(\phi_{t^{-1} u}^{-1}\left(y_{1}\right)\right)=\phi_{u u^{-1}}\left(\phi_{t}\left(y_{1}\right)\right)=\phi_{u u^{-1}}\left(x_{1}\right)=x_{1}$. Then $x_{1} \leq^{\prime} x_{3}$ in view of (27), which completes the proof of transitivity of $\leq^{\prime}$, and thus $\leq^{\prime}$ is a partial order.

Suppose that $x_{2} \in \operatorname{dom} \phi_{t}$ and $x_{1} \leq^{\prime} x_{2}$. By (24) there are $u \in T$ and $y_{1}, y_{2} \in \operatorname{dom} \phi_{u} \cap Y$, such that $x_{1}=\phi_{u}\left(y_{1}\right), x_{2}=\phi_{u}\left(y_{2}\right)$ and $y_{1} \leq y_{2}$. It follows that $y_{2} \in \operatorname{dom} \phi_{t u}=\operatorname{dom} \phi_{(t u)^{-1} t u}$. Since $\phi_{(t u)^{-1} t u}\left(y_{2}\right)=y_{2} \in Y$, we have that $y_{2} \in \operatorname{dom} \tau_{(t u)^{-1} t u}$. Then $\alpha\left(y_{2}\right) \leq(t u)^{-1} t u$. But $\alpha$, being a homomorphism of meet semilattices $Y \rightarrow E(T)$, is order-preserving, so $\alpha\left(y_{1}\right) \leq \alpha\left(y_{2}\right)$ in $E(T)$, and by transitivity $\alpha\left(y_{1}\right) \leq(t u)^{-1} t u$. Therefore, $\phi_{\alpha\left(y_{1}\right)} \leq \phi_{(t u)^{-1} t u}=\phi_{t u}^{-1} \phi_{t u}$. As $y_{1} \in \operatorname{dom} \tau_{\alpha\left(y_{1}\right)} \subseteq \operatorname{dom} \phi_{\alpha\left(y_{1}\right)}$, we conclude that $y_{1} \in \operatorname{dom}\left(\phi_{t u}^{-1} \phi_{t u}\right)=\operatorname{dom} \phi_{t u}=$ $\operatorname{dom}\left(\phi_{t} \phi_{u}\right)$. The latter implies that $x_{1}=\phi_{u}(y) \in \operatorname{dom} \phi_{t}$, so that dom $\phi_{t}$ is an order ideal of $\left(X, \leq^{\prime}\right)$. Notice also that in this case $\phi_{t}\left(x_{1}\right)=\phi_{t u}\left(y_{1}\right)$ and $\phi_{t}\left(x_{2}\right)=\phi_{t u}\left(y_{2}\right)$. Hence $\phi_{t}\left(x_{1}\right) \leq^{\prime} \phi_{t}\left(x_{2}\right)$, and $\phi_{t}$ is an order homomorphism dom $\phi_{t} \rightarrow \operatorname{ran} \phi_{t}$. Since the same holds for $\phi_{t^{-1}}=\phi_{t}^{-1}$, the bijection $\phi_{t}$ is an order isomorphism between order ideals of $\left(X, \leq^{\prime}\right)$.

Let $y_{1}, y_{2} \in Y$ with $y_{1} \leq y_{2}$. Since $y_{1}=\tau_{\alpha\left(y_{1}\right)}\left(y_{1}\right), y_{2}=\tau_{\alpha\left(y_{2}\right)}\left(y_{2}\right)$ and $\operatorname{ran} \tau_{\alpha\left(y_{1}\right)}$, $\operatorname{ran} \tau_{\alpha\left(y_{2}\right)}$ are ideals of $Y$, we have that $y_{1}, y_{2} \in \operatorname{ran} \tau_{\alpha\left(y_{1}\right)} \cap \operatorname{ran} \tau_{\alpha\left(y_{2}\right)} \subseteq \operatorname{ran} \phi_{\alpha\left(y_{1}\right)} \cap$ $\operatorname{ran} \phi_{\alpha\left(y_{2}\right)}=\operatorname{ran} \phi_{\alpha\left(y_{1} \wedge y_{2}\right)}$, whence $y_{1}=\phi_{\alpha\left(y_{1} \wedge y_{2}\right)}\left(y_{1}\right)$ and $y_{2}=\phi_{\alpha\left(y_{1} \wedge y_{2}\right)}\left(y_{2}\right)$. Therefore, $y_{1} \leq^{\prime} y_{2}$. Conversely, suppose that $y_{1}, y_{2} \in Y$ with $y_{1} \leq^{\prime} y_{2}$. By (24) there are $t \in T$ and $z_{1}, z_{2} \in Y \cap \operatorname{dom} \phi_{t}$, such that $y_{1}=\phi_{t}\left(z_{1}\right), y_{2}=\phi_{t}\left(z_{2}\right)$ and $z_{1} \leq z_{2}$. Observe that $y_{1}=\tau_{t}\left(z_{1}\right), y_{2}=\tau_{t}\left(z_{2}\right)$, and consequently $y_{1} \leq y_{2}$, as $\tau_{t}$ is order-preserving. This shows that the intersection of $\leq^{\prime}$ with $Y \times Y$ coincides with $\leq$. In particular, $\left(Y, \leq^{\prime}\right)=(Y, \leq)$ is a meet subsemilattice of $\left(X, \leq^{\prime}\right)$.

We now prove that $Y$ is an ideal of $X$. Suppose that $y \in Y$ and $x \leq^{\prime} y$ for some $x \in X$. There are $t \in T$ and $y_{1}, y_{2} \in Y \cap \operatorname{dom} \phi_{t}$ with $y_{1} \leq y_{2}$, such that $x=\phi_{t}\left(y_{1}\right)$ and $y=\phi_{t}\left(y_{2}\right)$. Then $y_{2}=\tau_{t}^{-1}(y)$, and since $\operatorname{dom} \tau_{t}$ is an ideal of $Y, y_{1} \in \operatorname{dom} \tau_{t}$, so that $x=\tau_{t}\left(y_{1}\right) \in Y$.

Proposition 4.4. Let $\tau$ be a globalizable strict partial action of an inverse semigroup $T$ on a semilattice $Y$ such that $\operatorname{dom} \tau_{t} \neq \emptyset$ for all $t \in T$. Then there exists a globalization $\phi: T \rightarrow \mathcal{I}(X), \iota: Y \rightarrow X$ of $\tau$ such that $(T, X, \iota(Y))$ is a strict $L$ triple. Moreover, in this case $Y \rtimes_{\tau} T \cong L(T, X, \iota(Y))$ and $Y \rtimes_{\tau}^{m} T \cong L_{m}(T, X, \iota(Y))$. In particular, if $\tau$ is fully strict, then $\operatorname{dom} \tau_{t} \neq \emptyset$ is automatically satisfied for all $t \in T$, and in this case $(T, X, \iota(Y))$ is also fully strict.

Proof. Existence of a pair $\phi: T \rightarrow \mathcal{I}(X), \iota: Y \rightarrow X$, such that $(T, X, \iota(Y))$ is an $L$-triple, follows from Lemmas 4.2 and 4.3 and the observation at the beginning of the proof of Proposition 4.1 (see (20)). The strictness of $(T, X, \iota(Y))$ is guaranteed by the strictness of $\tau$ : the corresponding homomorphism $e: \iota(Y) \rightarrow E(T)$ is just the (usual) composition of $\alpha$ with $\bar{\iota}^{-1}$. The isomorphism $Y \rtimes_{\tau} T \cong L(T, X, \iota(Y))$ 
is simply the identification of $(y, t) \in Y \rtimes_{\tau} T$ with $(\iota(y), t) \in L(T, X, \iota(Y))$, which restricts to the isomorphism $Y \rtimes_{\tau}^{m} T \cong L_{m}(T, X, \iota(Y))$. Finally, if $\tau$ is fully strict, then for every $t \in T$ there exists $y \in Y$, such that $(y, t) \in Y \rtimes_{\tau}^{m} T$. In particular, $y \in \operatorname{ran} \tau_{t}$, so that $\operatorname{dom} \tau_{t}=\operatorname{ran} \tau_{t^{-1}} \neq \emptyset$ for all $t \in T$. The full strictness of $(T, X, \iota(Y))$, whenever $\tau$ is fully strict, is immediate.

Propositions 4.1 and 4.4 permit us to conclude that O'Carroll's [12, Theorem $4]$ is equivalent to the version of our Theorem 3.4 where the partial action $\tau$ is additionally globalizable. The latter property, however, does not always hold for a partial action, as the following example shows.

Example 4.5. Let $S$ be the meet semilattice $\{0, e, f\}$, where 0 is the minimum element and $e \wedge f=0$. Consider the least congruence $\rho$ on $S$ which contains the pair $(0, e)$. Then $\rho$ is idempotent pure and the partial action $\tilde{\delta}$ of $S / \rho$ on $E(S)$ is not globalizable.

Proof. Indeed, the fact that $\rho$ is idempotent pure is trivial, since $S=E(S)$. Notice that the $\rho$-classes are $[e]=\{e, 0\}$ and $[f]=\{f\}$, so that $[e] \leq[f]$. Now,

$$
\begin{aligned}
& \tilde{\delta}_{[e]}=\delta_{e} \vee \delta_{0}=\operatorname{id}_{e E(S)} \vee \operatorname{id}_{0 E(S)}=\operatorname{id}_{\{0, e\}} \vee \operatorname{id}_{\{0\}}=\operatorname{id}_{\{0, e\}}, \\
& \tilde{\delta}_{[f]}=\delta_{f}=\operatorname{id}_{f E(S)}=\operatorname{id}_{\{0, f\}} .
\end{aligned}
$$

Since $\tilde{\delta}_{[e]} \not \tilde{\delta}_{[f]}$, the premorphism $\tilde{\delta}$ is not order-preserving. Thus, it is not globalizable as a partial action.

\section{ACKNOWLEDGEMENTS}

The author thanks the referee for the very detailed reading of the manuscript and numerous useful suggestions which permitted to simplify and shorten the proofs. In particular, the use of [6. Proposition 1.2.1] in the proofs of Lemmas 2.1 and 2.2. as well as the use of the order-preserving property of $\tau_{t}$ in the proof of Lemma 2.6 are due to the referee. Section 4 also arose from referee's comments.

\section{REFERENCES}

[1] Birget, J.-C., And Rhodes, J. Group theory via global semigroup theory. J. Algebra 120, 2 (1989), 284-300.

[2] Exel, R. Partial actions of groups and actions of inverse semigroups. Proc. Amer. Math. Soc. 126, 12 (1998), 3481-3494.

[3] Gould, V., AND Hollings, C. Partial actions of inverse and weakly left $E$-ample semigroups. J. Aust. Math. Soc. 86 (2009), 355-377.

[4] Kellendonk, J., and Lawson, M. V. Partial actions of groups. Internat. J. Algebra Comput. 14,1 (2004), 87-114.

[5] Lawson, M. V. A class of actions of inverse semigroups. J. Algebra 179, 2 (1996), 570-598.

[6] Lawson, M. V. Inverse semigroups. The theory of partial symmetries. World Scientific, Singapore-New Jersey-London-Hong Kong, 1998.

[7] Lawson, M. V., And Margolis, S. W. In McAlister's footsteps: a random ramble around the P-theorem. In Semigroups and formal languages. World Sci. Publ., Hackensack, NJ, 2007, pp. $145-163$.

[8] Lawson, M. V., Margolis, S. W., and Steinberg, B. Expansions of inverse semigroups. J. Aust. Math. Soc. 80, 2 (2006), 205-228.

[9] McAlister, D. B. Groups, semilattices and inverse semigroups II. Trans. Am. Math. Soc. 196 (1974), 351-370.

[10] O'Carroll, L. Inverse semigroups as extensions of semilattices. Glasgow Math. J. 16, 1 (1975), 12-21. 
[11] O'CARroll, L. Idempotent determined congruences on inverse semigroups. Semigroup Forum 12, 3 (1976), 233-243.

[12] O'Carroll, L. Strongly E-reflexive inverse semigroups. Proc. Edinburgh Math. Soc. (2) 20 (1977), 339-354.

[13] Petrich, M. Inverse semigroups. Pure and Applied Mathematics (New York). John Wiley \& Sons, Inc., New York, 1984. A Wiley-Interscience Publication.

[14] Steinberg, B. Inverse semigroup homomorphisms via partial group actions. Bull. Aust. Math. Soc. 64, 1 (2001), 157-168.

[15] Szendrei, M. B. A note on Birget-Rhodes expansion of groups. Journal of Pure and Applied Algebra 58, 1 (1989), 93-99.

Departamento de Matemática, Universidade Federal de Santa Catarina, Campus Reitor João David Ferreira Lima, Florianópolis, SC, CEP: 88040-900, Brazil

E-mail address: nskhripchenko@gmail.com 